\title{
Esquemas iniciais desadaptativos como mediadores entre os maus tratos na infância e a violência no namoro na adolescência
}

\author{
Early maladaptive schemas as mediators between child \\ maltreatment and dating violence in adolescence
}

Jeane Lessinger Borges (https://orcid.org/0000-0002-1925-7047) ${ }^{1}$

Débora Dalbosco Dell'Aglio (https://orcid.org/0000-0003-0149-6450) ${ }^{2}$

${ }^{1}$ Núcleo de Estudos e Pesquisa em Adolescência, Instituto de Psicologia, Universidade Federal do Rio Grande do Sul (UFRGS). R. Ramiro Barcelos 2600, Santa Cecília. 90035-003 Porto Alegre RS Brasil. jeanepsico07@gmail.com ${ }^{2}$ Programa de PósGraduação em Psicologia, UFRGS. Porto Alegre RS Brasil.

\begin{abstract}
This study investigated the association between exposure to child maltreatment and dating physical violence in the affective-sexual relationship among adolescents $(n=397,14-19$ years). A mediation model was conducted to determine whether these associations can be mediated by early maladaptive schemas (EMS), from the Schema Therapy's theoretical approach. Also, it sought to verify the invariant model by gender. The results showed that teen dating violence perpetrators with a history of child maltreatment had significantly higher scores in the perpetration of intimate violence than adolescents with no history of maltreatment. Disconnection and rejection realm schemas were mediators between exposure to child maltreatment and dating physical violence in adolescence, and this model was adequate to females. The clinical implications of these findings were also discussed.
\end{abstract}

Key words Childhood maltreatment, Intimate partner violence, Dating violence, Schema therapy
Resumo Este estudo investigou a associação entre a exposição aos maus tratos na infância e a perpetração de violência física nas relações afetivo-sexuais de adolescentes ( $n=397$; $14-19$ anos). Um modelo de mediação foi conduzido para determinar se tais relações podem ser mediadas por Esquemas Iniciais Desadaptativos (EIDs), a partir da abordagem teórica da Terapia dos Esquemas. Além disso, buscou-se verificar se o modelo é invariante para adolescentes do sexo feminino e masculino. Os resultados indicaram que adolescentes perpetradores de violência no namoro, com histórico de maus tratos na infância, tiveram escores significativamente mais altos na perpetração de violência intima, do que adolescentes sem histórico de maus tratos. Os EIDs do domínio de Desconexão e Rejeição foram considerados mediadores entre a exposição aos maus tratos e a violência no namoro na adolescência, sendo que este modelo se mostrou mais adequado ao sexo feminino. Implicações clínicas destes achados foram discutidas.

Palavras-chave Violência infantil, Violência por parceiro intimo, Violência no namoro, Terapia do Esquema 


\section{Introdução}

A violência no namoro (teen dating violence) engloba uma variedade de comportamentos abusivos, incluindo atos de violência física, psicológica e sexual, que ocorrem dentro de um contexto de relações românticas atuais ou passadas entre pré-adolescentes, adolescentes e jovens adultos ${ }^{1}$. Esse estudo busca dar ênfase à perpetração física nos relacionamentos do tipo "ficar" e namorar em adolescentes, investigada a partir do Conflict in Adolescent Dating Relationships Inventory $(\mathrm{CADRI})^{2}$. A categoria de violência física, nesse instrumento, engloba situações de agressões que incluem dar tapa, bater, chutar, dar soco, empurrar, puxar o cabelo ou usar objetos para ferir o outro (jogar algo contra a pessoa).

No que se refere à violência física no namoro, estudos indicam que sua prevalência é alta e diversificada em diferentes países, conforme o método e a concepção de violência adotada. A prevalência variou entre 5,9\% em Portugal ${ }^{3}, 14,8 \%$ na Coréia do Sul ${ }^{4}$ e $24,3 \%$ na Espanha ${ }^{5}$. No estudo multicêntrico internacional desenvolvido por Straus ${ }^{6}$, incluindo 31 universidades em 16 países, houve uma taxa de $29 \%$ de perpetração física em relacionamentos de jovens universitários. Estudo de revisão sistemática da literatura indicou que $25 \%$ das meninas e $13 \%$ dos meninos (idades entre 13 e 18 anos) perpetraram violência física em seus relacionamentos íntimos ${ }^{7}$. Para as pesquisas que utilizaram a CADRI como medida de investigação da perpetração física no namoro, foi observada uma prevalência similar em alguns países. Por exemplo, estudo com 918 adolescentes, nos EUA, indicou que $19,8 \%$ perpetraram violência física ${ }^{8}$. Em outro estudo com 729 jovens universitários, no México, a perpetração física foi de $16,6 \% 9$. No Brasil, estudo multicêntrico com 3.205 adolescentes de 15 a 19 anos, de 10 capitais, indicou uma taxa de perpetração de $24,1 \%{ }^{10}$.

Estudos prévios indicam que a exposição aos maus tratos na infância é um importante fator de risco à ocorrência de violência no namoro na adolescência ${ }^{11-14}$. Um estudo com 2.541 jovens universitários (EUA) indicou que 26,5\% dos adolescentes com histórico de abuso infantil perpetraram violência em seus relacionamentos íntimos ${ }^{15}$. Em outro estudo com jovens da Coréia do Sul, vítimas de maus tratos físicos na infância, houve um aumento de 2,11 na chance de ser perpetrador de violência no namoro, quando comparadas a jovens que não sofreram maus tratos ${ }^{4}$.

Os maus tratos têm sido descritos como parte do fenômeno de transmissão intergeracional da violência, no qual crianças expostas a esses tipos de estressores no contexto familiar experienciam e perpetram violência quando adultas, repetindo os padrões de interação interpessoal aprendidas na infância ${ }^{15}$. Estudos têm adotadoa Teoria da Apredizagem Social ${ }^{16}$ para explicar as relações entre essas variáveis.Contudo, limitações foram apontadas nestes estudos, uma vez que nem todos os adolescente expostos à violência intrafamiliar perpetram violência em suas relações íntimas $^{17}$.

Estudos atuais têm indicado que o efeito da exposição aos maus tratos na infância não seria direto, mas que haveria diferentes variáveis cognitivas e emocionais que funcionariam como mediadoras dessa relação. Por exemplo, crenças sobre aceitação da violência ${ }^{18}$, regulação da raiva e sintomas psicológicos derivados do trauma infantil $^{19}$ foram descritos como variáveis mediadoras ${ }^{14,20}$. Em um estudo com adolescentes espanhóis, a violência na família foi associada com perpetração de violência no namoro e parte dessa associação foi mediada por crenças que justificam a violência no namoro e por esquemas cognitivos de grandiosidade ${ }^{21}$. Já em um estudo com adolescentes mexicanos ${ }^{19}$, a falta de controle da raiva e uma maior aceitação quanto ao uso de violência mediaram as relações entre conflito interparental e perpetração de violência no namoro.

Estudo com jovens coreanos indicou que tanto meninos quanto meninas expostos aos maus tratos na infância têm risco maior para se tornar perpetradores de violência no namoro ${ }^{22}$. Contudo, há resultados que apontam que a variável sexo deve ser controlada. Por exemplo, meninos que sofreram maus tratos infantis desenvolveram maior atitute de aceitação à violência, enquanto que meninas desenvolveram déficits na capacidade de empatia ${ }^{14}$. Portanto, a forma como os maus tratos influenciam na predição de violência no namoro em adolescentes é mediada por diferentes fatores e por sexo, o que justifica averiguar a invariância do modelo.

A fim de avançar na compreensão de como a violência intrafamiliar na infância pode contribuir para a perpetração da violência no namoro, busca-se neste estudo adotar os pressupostos teóricos da Terapia dos Esquemas ${ }^{23,24}$. Nesta teoria, os Esquemas Iniciais Desadaptativos (EIDs) assumem um papel importante, os quais podem ser compreendidos como padrões emocionais e cognitivos autoderrotistas, resistentes à mudança, disfuncionais em um grau significativo, e associados à visão negativa de si em relação ao 
ambiente $^{23}$. Os EIDs desenvolvem-se durante a infância e são elaborados ao longo da vida, sendo o resultado do temperamento inato da criança interagindo com as experiências iniciais com os pais e cuidadores. Nas fases posteriores do ciclo vital, os esquemas são ativados por acontecimentos ambientais relevantes para o esquema, como, por exemplo, frente aos conflitos nos relacionamentos interpessoais do indivíduo ${ }^{24}$. Necessidades emocionais básicas não atendidas na infância precoce e padrões continuados de experiências nocivas, como maus tratos infantis, são descritas como as principais origens do desenvolvimento dos EIDs ${ }^{23-25}$. Estudos têm indicado uma associação significativa entre maus tratos na infância e $\operatorname{EIDs}^{26,27}$.

De acordo com a Terapia dos Esquemas, 18 EIDs foram identificados, os quais são agrupados em cinco domínios de esquemas, correspondendo às necessidades desenvolvimentais da criança ${ }^{25}$. O primeiro domínio é nomeado de Desconexão e Rejeição e está associado à necessidade básica de segurança, estabilidade emocional, cuidado, sentimento de conexão e de aceitação. Quando essas necessidades emocionais básicas não são atendidas satisfatoriamente, o indivíduo fica propenso a desenvolver os esquemas de Abandono/instabilidade, Desconexão/abuso, Privação emocional, Defectividade/vergonha e Isolamento social/alienação. O segundo domínio é o de Autonomia e Desempenho Prejudicados. A família de origem tende a ser superprotetora, emaranhada e com dificuldade em desenvolver a autonomia da criança ${ }^{24}$. As pessoas com esquemas deste domínio tendem a ter dificuldade em se perceber como autônomos e independentes ${ }^{25}$. Os EIDs de Dependência/incompetência, Vulnerabilidade ao Dano, Emaranhamento/self subdesenvolvido e Fracasso compõem este domínio. O terceiro domínio é o de Limites Prejudicados, caracterizado pela falta de limites internos, dificuldade em reconhecer os direitos alheios e em estabelecer compromissos ou definir e cumprir objetivos pessoais realistas ${ }^{28}$. A família típica é permissiva, indulgente ou exageradamente crítica e punitiva ${ }^{25}$. Os EIDs deste domínio são o de Arrogo/grandiosidade e o de Autocontrole/autodisciplina insuficientes. O quarto domínio é o de Direcionamento para o Outro, caracterizado pela preocupação excessiva com as necessidades alheias, à custa das próprias necessidades ${ }^{24}$. Os EIDs pertencentes a esse domínio são: Subjugação, Autossacrifício e Busca por Aprovação. O quinto domínio é nomeado de Supervigilância e Inibição e está associado ao cumprimento de regras rígidas internalizadas, à custa da felicidade e da expressão espontânea de sentimentos. A família típica é severa, punitiva e rígida ${ }^{24}$. Os EIDs desse domínio são: Negativismo/pessimismo, Inibição emocional, Padrões inflexíveis/postura crítica exagerada e Postura punitiva.

Pesquisas têm apontado a presença dos esquemas do domínio de Desconexão e Rejeição em adolescentes e mulheres adultas vítimas de maus tratos na infância e de violência na intimidade. Na população de adolescentes, associação entre violência na família e perpetração de violência no namoro foi observada em um estudo longitudinal com adolescentes espanhóis, indicando que os EIDs dos domínios de Desconexão e Rejeição podem atuar como mecanismos pelos quais a violência na infância pode ser transmitida intergeracionalmente ${ }^{28}$. Na população adulta, estudos prévios têm apontado associação entre violência do parceiro íntimo e EIDs em mulheres adultas, sobretudo no que se refere ao papel dos esquemas do domínio de Desconexão e Rejeição ${ }^{29-31}$. Esses diferentes estudos indicam que os EIDs podem ser um dos fatores cognitivos associados à interação entre violência na infância e violência na intimidade.

No Brasil, poucos estudos têm investigado o perfil de EIDs em adultos com histórico de violência conjugal ${ }^{30,32}$. Os esquemas de desconfiança/ abuso, defectividade/vergonha, isolamento social foram significativamente associados ao grupo de adultos com histórico de violência conjugal, quando comparados a outro grupo sem violência conjugal $^{30}$. Em outro estudo, com adultos de 22 a 74 anos, os esquemas de desconfiança/abuso, dependência/incompetência, emaranhamento e grandiosidade/arrogo foram relacionados com perpetração de violência conjugal ${ }^{32}$.

Ainda, no contexto brasileiro, estudos têm apontado a associação entre violência intrafamiliar e perpetração de violência física e psicológica no namoro de adolescentes ${ }^{33,34}$. Contudo, nesses estudos não há uma investigação sobre quais variáveis cognitivas podem influenciar a perpetração da violência no namoro em adolescentes. $\mathrm{O}$ objetivo deste estudo foi investigar a associação entre os EIDs e a perpetração de violência física no namoro em adolescentes. A hipótese proposta é de que a exposição aos maus-tratos na infância contribui para a ocorrência de violência física no namoro na adolescência, sendo que os EIDs podem assumir o papel de mediadores nessa associação. Buscou-se ainda investigar se o modelo é invariante para adolescentes do sexo feminino e masculino. Além disso, foram investigados os pa- 
drões de perpetração de violência no namoro por sexo e pela presença de maus tratos ao longo da infância. Diferença nos escores dos EIDs também foi investigada quanto à presença ou ausência de histórico de maus tratos na infância.

\section{Métodos}

\section{Participantes}

Participaram deste estudo transversal e retrospectivo, 525 adolescentes (58,5\% feminino), entre 14 e 19 anos de idade. A coleta de dados ocorreu em oito escolas públicas e duas privadas, de Ensino Médio, das cidades de Porto Alegre/RS e de Novo Hamburgo/RS. A maioria das escolas eram públicas $(66,8 \%)$, duas escolas eram privadas $(14,9 \%)$ e uma escola era profissionalizante $(18,3 \%)$. Foram utilizados como critérios de inclusão: ser estudante de Ensino Médio; ter entre 14 e 19 anos de idade(considerando que nesta etapa da adolescência há uma frequência maior de relacionamentos amorosos entre os jovens); e já ter tido ou estar em um relacionamento amoroso na adolescência ("ficar" ou namorar).

\section{Instrumentos}

Foram utilizados os seguintes instrumentos de autorrelato:

Questionário de Dados sociodemográfios: para avaliar características individuais (idade, sexo, escolaridade, uso de álcool e outras drogas), familiares (com quem morava, presença de violência conjugal entre os pais, uso de drogas por familiares) e sobre os relacionamentos afetivo-sexuais atuais ou passados.

Inventário de Conflitos nas Relações de $\mathrm{Na}$ moro na Adolescência (CADRI) ${ }^{2,35}$ : que avalia a presença e a frequência de comportamentos abusivos em relacionamentos afetivo-sexuais na adolescência. $\mathrm{O}$ instrumento é composto por 70 questões e é respondido em uma escala Likert de quatro pontos, variando de 0 (nunca) a 3 (sempre, mais de seis vezes). A CADRI investiga as seguintes categorias de violência: Violência Física (4 itens), Violência Psicológica verbal/emocional (10 itens), Violência Psicológica/Ameaças (4 itens), Violência Psicológica Relacional (3 itens), e Violência Sexual (4 itens). Neste estudo foi utilizado o somatório total dos itens da sub-escala de perpetração de violência no namoro. No estudo da versão brasileira ${ }^{35}$, o Alpha para a violência sofrida foi de 0,87 e para a violência per- petrada foi de 0,88 . Na pesquisa atual, os Alphas de Cronbach variaram entre 0,87 para violência perpetrada e 0,90 para violência sofrida.

Questionário de Esquemas para Adolescentes $(\mathrm{QEA})^{36,37}$ : versão luso-brasileira, que avalia os 18 esquemas iniciais desadaptativos, por meio de 52 itens, apresentados de forma não consecutiva, em uma escala Likert de seis pontos ( 1 = Não tem nada a ver com o que acontece comigo a $6=\hat{E}$ exatamente o que acontece comigo). O escore total de cada EID é calculado pela média do somatório dos itens que compõem o esquema em questão. $\mathrm{Na}$ amostra brasileira, a consistência interna variou entre $\alpha=$ .63 para o esquema de Arrogo/Grandiosidade e $\alpha$ $=.88$ para o esquema de Fracasso ${ }^{37}$.

Inventário de Exposição à Violência Intrafamiliar na Infância (IEVII) ${ }^{37}$ : este instrumento foi desenvolvido pelas autoras a fim de investigar, de forma retrospectiva, a exposição dos adolescentes a situações de maus tratos ao longo da infância, perpetrado pelos pais ou cuidadores principais. O IEVII é composto por 19 itens, que são respondidos por meio de uma escala Likert de quatro pontos $(0=$ nunca e $3=$ sempre $)$, que avaliam quatro tipos de maus tratos infantis: 1 ) Abuso físico; 2) Abuso psicológico/abandono; 3 ) Abuso sexual; e 4) Negligência. Os itens foram criados a partir da literatura referente aos maus tratos infantis ${ }^{38,39}$, sendo que três profissionais experts na área foram convidados para ser juízes. Posteriormente, foi realizado um estudo piloto com 15 adolescentes de uma escola pública, a fim de avaliar o conteúdo semântico dos itens para o público adolescente. Na pesquisa atual, o Alpha da escala geral foi de 0,84 .

\section{Procedimentos}

Este estudo seguiu as diretrizes éticas para pesquisa com seres humanos, conforme a Resolução 510/2016 do Conselho Nacional de Saúde. A aplicação foi coletiva, nas próprias escolas, com duração média de uma hora. A participação dos adolescentes na pesquisa ocorreu de forma voluntária, após rapport inicial em que foram apresentados os objetivos do estudo. Foi solicitado o Termo de Consentimento Livre e Esclarecido (para os pais dos adolescentes menores de 18 anos e para os próprios adolescentes com idade acima de 18 anos), assim como o assentimento dos adolescentes. Nas sessões de coleta de dados, um membro da equipe de pesquisa auxiliou no preenchimento dos questionários. Este estudo foi aprovado pelo Comitê de Ética em Psicologia da Universidade Federal do Rio Grande do Sul. 


\section{Análises de dados}

Inicialmente foi realizada uma descrição dos padrões de perpetração de violência no namoro. Foram considerados perpetradores os adolescentes que tiveram pontuação maior ou igual a um ponto nos escores da CADRI perpetração. Para o grupo de exposição aos maus tratos na infância foram considerados os adolescentes que obtiveram escore maior ou igual a um ponto no escore total do IEVII. A partir desses resultados, a amostra foi dividida em três grupos: G1 - perpetradores de violência no namoro e vítimas de maus-tratos na infância; G2 - perpetradores de violência no namoro e não vítimas de maustratos na infância; e G3 - não perpetradores de violência no namoro. Foi realizada análise de frequência simples dos maus tratos sofridos na infância, por sexo, para adolescentes do G1. Foi realizado teste $t$ para verificar diferença nos escores da CADRI por grupo (G1 e G2) e para investigar diferença por sexo nos escores do IEVII. Nesse estudo, foi utilizado intervalo de confiança de $95 \%$ e cálculo do tamanho de efeito, sendo que a magnitude foi classificada em: 0,20-0,49= pequena; $0,50-0,79=$ moderada; e acima de 0,80 $=$ grande $^{40}$.

No que se refere aos EIDs, foi realizada uma ANOVA, seguida de teste post hoc de Tukey, para investigar diferença nos escores dos EIDs entre os grupos (G1, G2 e G3). Foram utilizados os escores médios dos itens de cada EIDs para o cálculo da média (pontos ponderados). Além disso, foi realizado um teste $t$ para examinar se há diferença por sexo nos EIDs.

Para o modelo de mediação ${ }^{41}$, foi utilizado o escore total do IEVII, o escore da CADRI perpetração física e o somatório dos escores ponderados dos EIDs do domínio de Desconexão e Rejeição. Assim, buscou-se testar um modelo de mediação em que a exposição aos maus tratos na infância (variável independente X) afeta direta e indiretamente a perpetração de violência nos relacionamentos afetivo-sexuais na adolescência (variável dependente Y), sendo que essa relação pode ser mediada pela presença de EIDs do domínio de Desconexão e Rejeição (variável mediadora M). Foram testados vários modelos (com os cinco domínios propostos por Jeffrey Young ${ }^{23,24}$ ), sendo que o único modelo de mediação significativo e com ajuste adequado foi o que incluiu os maus-tratos na infância, a perpetração de violência física no namoro e os EIDs de Abandono/instabilidade, Desconfiança/abuso, Privação emocional, Defectividade/vergonha e Isolamento social, que teoricamente, compõem o domínio de Desconexão e Rejeição (Variável mediadora). A escolha deste modelo foi ainda embasada teoricamente, a partir dos estudos internacionais sobre a associação entre maus tratos na infância, os EIDs de Desconexão e Rejeição e a violência na intimidade ${ }^{28,29}$.

O modelo de mediação foi realizado com o pacote Lavaan $^{42}$, com estimador Maximum Likelihood (ML) no ambiente $\mathrm{R}$ ( $R$ Development Core Team $)^{43}$. Os indicadores de ajuste do modelo utilizados foram Comparative Fit Index (CFI), Tucker-Lewis Index (TLI), Root Mean Square of Approximation (RMSEA), e o Standardized Root Mean Square Residual (SRMR). O modelo é ajustado quando TLI e CFI apresentam valores acima de 0,90, RMSEA, valores abaixo de 0,08 e SRMR, valores abaixo de $0,10^{44}$. Foi ainda realizada uma análise de múltiplos grupos a fim de investigar a invariância do modelo, por sexo.

\section{Resultados}

Da amostra geral $(n=525), 75,62 \%(n=397)$ dos adolescentes foram caracterizados como perpetradores de violência nas relações afetivosexuais na adolescência, sendo que destes $60,70 \%$ $(n=241)$ eram do sexo feminino e $39,30 \%$ ( $n=$ 156) do sexo masculino. Foram, então, compostos três grupos, de acordo com os escores nos instrumentos (CADRI e IEVII), conforme descritos a seguir:

Grupo 1 (G1) - Adolescentes perpetradores de violência nas relações afetivo-sexuais na adolescência e vitimas de maus tratos na infância: 288 adolescentes ( $n=167$ sexo feminino, $58,0 \% ; n=$ 121 sexo masculino, 42,0\%), com idade média de 16,71 anos $(D P=1,14)$. No que se refere ao tipo de relacionamento afetivo-sexual vivenciado no momento da pesquisa, $67 \%$ dos adolescentes estavam namorando e 31,9\% "ficando" com alguém. Neste grupo $100 \%$ dos participantes relataram ter sido vítima de violência psicológica/ ameaças, $89,6 \%$ de violência física, $46,2 \%$ de negligência e $6,9 \%$ de abuso sexual. Cerca de 58,8\% relataram testemunhar agressões verbais entre os pais, 7,5\% testemunharam violência física e 5,4\% ameaças.

Grupo 2 (G2) - Adolescentes perpetradores de violência nas relações afetivo-sexuais na adolescência e não vítimas de maus tratos na infância: 109 adolescentes $(n=74$ sexo feminino, $67,9 \% ; n=$ 35 sexo masculino, $32,1 \%$ ), com média de idade de 16,54 anos $(D P=1,30)$. No momento da co- 
leta de dados, a maioria dos adolescentes estava namorando $(73,1 \%)$ e $26,9 \%$ deles tinham um relacionamento do tipo "ficar".

Grupo 3 (G3) - Adolescentes não perpetradores de violência nas relações afetivo-sexuais na adolescência: 128 adolescentes $(n=66$ sexo feminino, $51,6 \% ; n=62$ sexo masculino, $48,4 \%$ ), com idade média de 16,52 anos $(D P=1,20)$. Neste grupo, adolescentes também foram expostos aos maustratos na infância, mas em uma frequência menor do que os adolescentes do G1. Assim, 81,3\% relataram ter sofrido violência psicológica, $71,10 \%$ violência física, $28,0 \%$ negligência e 3,0\% abuso sexual. No momento da pesquisa, $60,4 \%$ dos adolescentes relataram estar namorando e 39,6\% de estar "ficando" com alguém.

A Tabela 1 descreve os padrões de perpetração de violência nas relações afetivo-sexuais, conforme as médias na CADRI, para G1 e G2 (com e sem histórico de maus-tratos na infância). De modo geral, houve diferença entre os grupos, embora com tamanhos de efeito pequenos, indicando que adolescentes do G1 perpetraram taxas mais elevadas de violência em suas relações afetivo-sexuais, quando comparados aos adolescentes do G2, com exceção da violência relacional.

Não foi observada diferença significativa nos escores do IEEVII, por sexo, para o grupo de adolescentes perpetradores de violêcia no namoro (G1, Figura 1).

Os resultados da ANOVA indicaram a presença de diferença significativa entre os três grupos nos escores dos EIDs (Tabela 2). O teste post hoc de Tukey indicou que houve diferença significativa no EID de Abandono entre o G1 e o G3 $(p=0,024)$. No EID de Desconfiança/abuso houve diferença significativa entre G1 e G3 ( $p=$ $0,001)$ e entre G1 e o G2 $(p=0,01)$. Houve apenas diferença significativa entre G1 e G2 para o
EID de Defectividade/vergonha $(p=0,033)$, de Isolamento social $(p=0,043)$ e de Privação emocional $(p=0,004)$.

Para o EID de Arrogo/grandiosidade houve diferença significativa entre G1 e G2 $(p=0,014)$ e entre G1 e G3 ( $p=0,001)$. Para os EIDs de Autocontrole insuficiente $(p=0,05)$ e de Padrões inflexíveis $(p=0,024)$ houve diferença apenas entre G1 e G2. No EID de Busca por aprovação houve diferença entre G1 e G2 $(p=0,013)$ e entre G1 e $\mathrm{G} 3(p=0,004)$. Finalmente, para o EID de Negativismo/pessimismo houve diferença entre G1 e G2 $(p=0,005)$ e entre G1 e G3 $(p=0,001)$. De modo geral, estes resultados indicam que os adolescentes perpetradores com histórico de maus tratos tendem a ter médias significativamente mais altas nos EIDs de Abandono, Desconfiança/ abuso, Defectividade/vergonha, Isolamento social, Privação emocional, Arrogo/grandiosidade, Padrões inflexíveis, Busca por aprovação e Negativismo/pessimismo.

Foi investigada diferença nos escores dos EIDs, por sexo (G1). Adolescentes do sexo feminino apresentaram escores significativamente mais altos no EID de Abandono $(M=4,40, D P$ $=1,33, \mathrm{t}=3,93, d f=395, \mathrm{p}=0,001)$ do que os adolescentes do sexo masculino $(M=3,86, D P$ $=1,30)$. Na mesma direção, adolescentes do sexo feminino tiveram média mais alta no EID de Desconfiança/abuso $(M=3,44, D P=1,35, \mathrm{t}=$ $2,14, d f=395, \mathrm{p}=0,033)$ do que os adolescentes do sexo masculino $(M=3,14, D P=1,34)$. Nos demais EIDs não houve diferença significativa.

O modelo teórico hipoteticamente elaborado para este estudo foi confirmado pelo modelo de mediação (Figura 2). Os maus tratos na infância (VI) foram significativamente associados aos EIDs do domínio Desconexão e Rejeição (VM), $B=0,39 ; z=9,639 ; p=0,001$. Por sua vez, os

Tabela 1. Médias e desvio-padrão de perpetração de violência no namoro, por grupo de adolescentes com ou sem histórico de maus tratos.

\begin{tabular}{|c|c|c|c|c|c|}
\hline \multirow{2}{*}{ Categoria de violência } & Grupo $1(n=288)$ & Grupo $2(n=109)$ & \multirow{2}{*}{$\mathbf{t}$} & \multirow{2}{*}{$\mathbf{P}$} & \multirow{2}{*}{ d } \\
\hline & M/DP & M/DP & & & \\
\hline Violência verbal/emocional & $7,00(4,76)$ & $5,36(4,27)$ & 3,14 & 0,002 & 0,35 \\
\hline Violência física & $0,84(1,79)$ & $0,50(1,28)$ & 2,08 & 0,040 & 0,20 \\
\hline Violência sexual & $0,79(1,13)$ & $0,53(0,94)$ & 2,32 & 0,020 & 0,24 \\
\hline Violência psicológica/ameaças & $0,68(1,44)$ & $0,20(0,56)$ & 4,81 & 0,001 & 0,38 \\
\hline Violência relacional & $0,24(0,76)$ & $0,21(0,79)$ & 0,29 & 0,776 & 0,04 \\
\hline CADRI perpetração total & $9,55(7,67)$ & $6,81(6,24)$ & 3,66 & 0,001 & 0,37 \\
\hline
\end{tabular}

Nota: CADRI = Inventário de Conflitos nas Relações de Namoro na Adolescência; $\mathrm{M}=$ média; $\mathrm{DP}=$ desvio-padrão; $t=$ teste $t ; p$ = nível de significância; $d=$ tamanho de efeito; $\mathrm{G} 1$ = adolescentes perpetradores com histórico de maus tratos na infância; G2 = adolescentes perpetradores sem histórico de maus tratos na infância. 


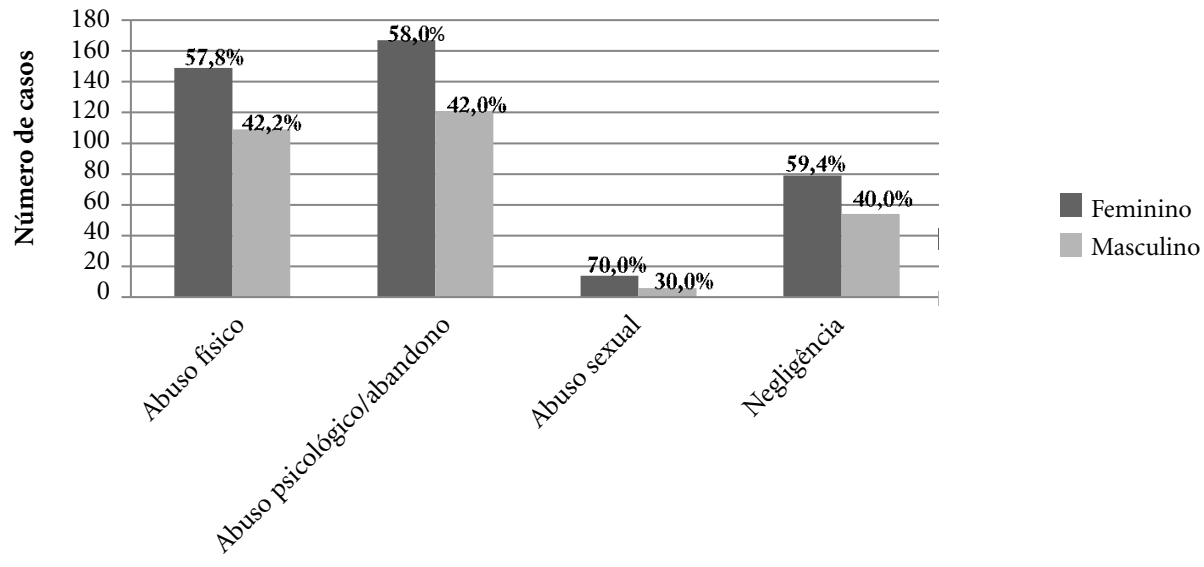

Categoria de maus tratos

Figura 1. Frequência de maus tratos, por sexo, em adolescentes perpetradores de violência no namoro (G1).

Tabela 2. Escores médios dos EIDs, por grupo $(n=525)$

\begin{tabular}{|c|c|c|c|c|c|}
\hline \multirow{2}{*}{ EIDs } & G1 $(n=288)$ & $\mathrm{G} 2(\mathrm{n}=128)$ & G3 $(n=129)$ & \multirow{2}{*}{ ANOVA } & \multirow{2}{*}{$\mathbf{p}$} \\
\hline & M/DP & M/DP & M/DP & & \\
\hline Abandono/instabilidade & $4,20(1,37)$ & $4,16(1,28)$ & $3,82(1,39)$ & $\mathrm{F}=3,60$ & 0,028 \\
\hline Desconfiança/abuso & $3,46(1,36)$ & $2,94(1,25)$ & $2,67(1,17)$ & $\mathrm{F}=18,46$ & 0,001 \\
\hline Privação emocional & $2,15(1,34)$ & $1,69(1,07)$ & $2,02(1,34)$ & $\mathrm{F}=5,15$ & 0,006 \\
\hline Defectividade/vergonha & $1,77(1,06)$ & $1,47(0,88)$ & $1,76(1,15)$ & $\mathrm{F}=3,39$ & 0,034 \\
\hline Isolamento social & $2,33(1,38)$ & $1,96(1,20)$ & $2,17(1,49)$ & $\mathrm{F}=3,00$ & 0,050 \\
\hline Dependência/incompetência & $1,68(0,90)$ & $1,54(0,85)$ & $1,66(0,82)$ & $\mathrm{F}=1,06$ & 0,347 \\
\hline Vulnerabilidade ao dano & $2,66(1,41)$ & $2,48(1,21)$ & $2,49(1,39)$ & $\mathrm{F}=0,98$ & 0,374 \\
\hline Emaranhamento & $2,76(1,41)$ & $3,38(1,44)$ & $2,92(1,40)$ & $\mathrm{F}=7,56$ & 0,001 \\
\hline Fracasso & $2,16(1,25)$ & $1,83(1,24)$ & $2,12(1,25)$ & $\mathrm{F}=2.77$ & 0,064 \\
\hline Arrogo/grandiosidade & $2,17(1,06)$ & $1,86(0,91)$ & $1,75(0,82)$ & $\mathrm{F}=9,79$ & 0,001 \\
\hline Autocontrole insuficiente & $2,42(1,18)$ & $2,02(0,96)$ & $2,19(1,16)$ & $\mathrm{F}=5,54$ & 0,004 \\
\hline Subjugação & $1,89(0,95)$ & $1,75(0,86)$ & $1,88-0,97$ & $\mathrm{~F}=1,02$ & 0,361 \\
\hline Autossacrifício & $3,77(1,27)$ & $3,50(1,27)$ & $3,48(1,31)$ & $\mathrm{F}=3,07$ & 0,060 \\
\hline Busca por aprovação & $2,93(1,36)$ & $2,54(1,17)$ & $2,48(1,28)$ & $\mathrm{F}=7,23$ & 0,001 \\
\hline Negativisto/pessimismo & $3,32(1,43)$ & $2,81(1,42)$ & $2,70(1,45)$ & $\mathrm{F}=10,43$ & 0,001 \\
\hline Inibição Emocional & $3,07(1,30)$ & $2,73(1,36)$ & $3,01(1,46)$ & $\mathrm{F}=2,40$ & 0,084 \\
\hline Padrões inflexíveis & $3,56(1,48)$ & $3,13(1,26)$ & $3,24(1,45)$ & $\mathrm{F}=4,43$ & 0,012 \\
\hline Postura punitiva & $2,31(1,26)$ & $2,19(1,30)$ & $2,16(1,14)$ & $\mathrm{F}=0,78$ & 0,458 \\
\hline
\end{tabular}

Nota. EID = Esquemas iniciais desadaptativos; $\mathrm{M}=$ média; $\mathrm{DP}=$ desvio-padrão; $\mathrm{F}=$ resultado da Anova; $p=$ nível de significância; G1 = adolescentes perpetradores com histórico de maus tratos na infância; $G 2=$ adolescentes perpetradores sem histórico de maus tratos na infância; G3 = adolescentes não perpetradores de violência no namoro.

EIDs (VM) foram significativamente associados à perpetração de violência física no namoro (VD), $B=0,17 ; z=3,566 ; p=0,001$. Este efeito indireto explica $15 \%$ da variância da perpetração da violência física. O efeito direto total dos maus tratos (VI) sobre a perpetração física no namoro $(\mathrm{VD})$ foi $\mathrm{ab}=0,064$ (Erro padrão $=0,02$ ). $\mathrm{A}$ relação entre os maus tratos e a perpetração de violência física perdeu então sua significância ( $B$ $=0,08 ; \mathrm{z}=1,762 ; p=0,08)$. Os indicadores de 


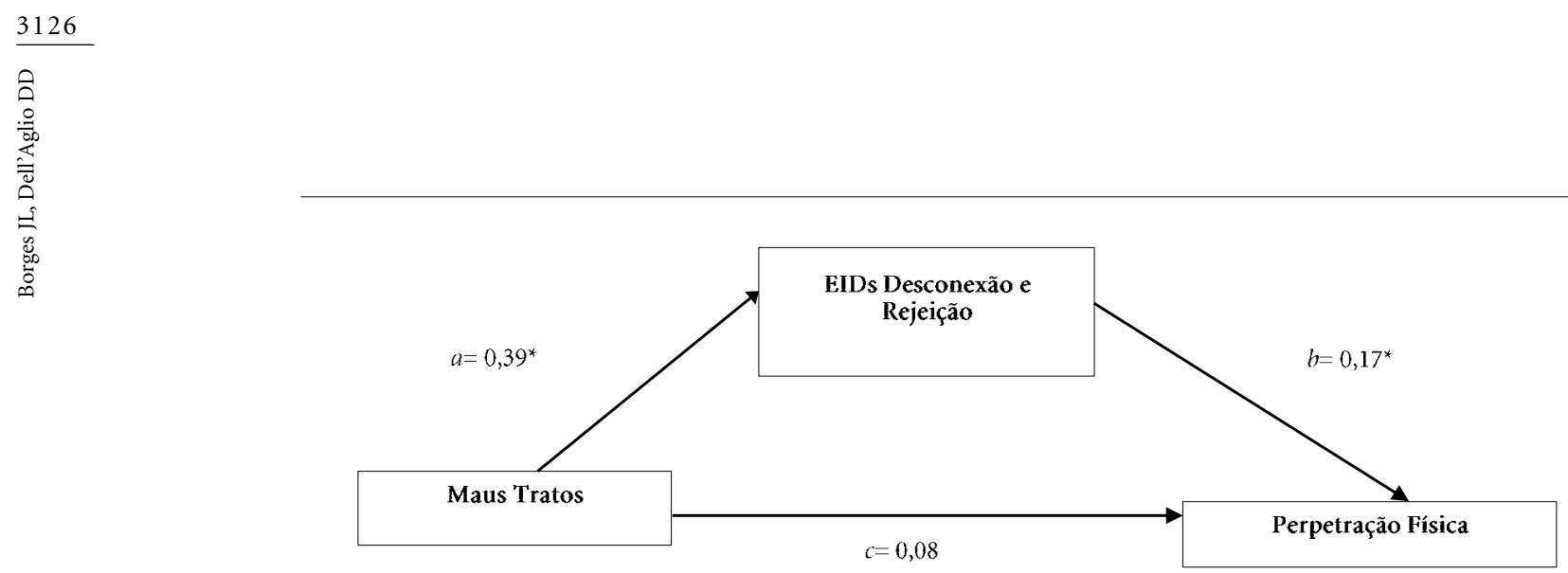

Figura 2. Modelo de mediação entre a exposição aos maus tratos na infância e a perpetração de violência física no namoro na adolescência.

Nota: ${ }^{*} \mathrm{p}<0,001$.

ajustamento global do modelo foram considerados adequados: $\chi^{2}=109.362 ; p=0,001$; CFI $=$ 1,00 ; TLI $=1,00$; RMSEA $=0,01$ (90\% IC, $\mathrm{p}<$ $0,05)$ e $\mathrm{SRMR}=0,00$.

Em seguida, foi realizada uma análise de mútiplos grupos simultânea para verificar se o modelo teórico é invariante entre o sexo feminino e masculino. Para a amostra das adolescentes, os maus tratos $(\mathrm{X})$ foram igualmente associados aos esquemas do domínio Desconexão e Rejeição $(\mathrm{M}), B=0,39 ; \mathrm{z}=7,472 ; p=0,001$. A variável mediadora (EIDs) foi significativamente associada à perpetração física no namoro $(\mathrm{Y}), \beta=0,21 ; \mathrm{z}=$ 3,$585 ; p=0,001$. O efeito direto entre os maus tratos e a perpetração de violência física foi de $B$ $=0,08 ; \mathrm{z}=1,418 ; p=0,10 . \mathrm{Na}$ amostra dos adolescentes do sexo masculino, novamente houve uma relação significativa entre a exposição aos maus tratos e os EIDs de Desconexão e Rejeição, $B=0,39 ; z=6,193 ; p=0,001$. Contudo, os EIDs do domínio de Desconexão e Rejeição não foram significativamente associados à perpetração física, $B=0,05 ; z=0,671 ; p=0,50$. A relação entre maus tratos e perpetração física foi de $B=0,11$; $\mathrm{z}=1,476 ; p=0,14$. Dessa forma, o modelo de mediação indica que o papel dos EIDs como mediador da relação entre a exposição aos maus tratos na infância e perpetração de violência física no namoro é relevante para as meninas, mas não para os meninos. Este modelo simultâneo indicou índices de ajustes adequados: $\chi 2=112.093$; $p=0,001 ; \mathrm{CFI}=1,00 ; \mathrm{TLI}=1,00 ; \mathrm{RMSEA}=0,01$ $(90 \%$ IC, $p<0,05)$ e SRMR $=0,00$.

\section{Discussão}

Este estudo examinou o papel dos EIDs como mediadores da exposição aos maus tratos na infância e a perpetração de violência física no namoro na adolescência. Adolescentes perpetradores com histórico de maus tratos tiveram escores significativamente mais altos na CADRI do que aqueles sem histórico de maus tratos. Esse resultado evidencia, de um lado, que os maus tratos na infância podem ser considerados um fator de risco para um modelo intergeracional de violência; por outro lado, revela ainda que outros fatores contextuais estão associados à perpetração de violência no namoro, uma vez que os adolescentes do G2, que não sofreram maus tratos na infância, igualmente perpetraram violência nas suas relações afetivo-sexuais. Há um consenso na literatura quanto à intergeracionalidade da violência nos casos de violência no namoro na adolescência ${ }^{11-13,15,20}$.

Os resultados indicaram ainda que adolescentes perpetradores com histórico de maus tratos tiveram escores mais altos nos EIDs, se comparados aos demais grupos. Um dos pressupostos básicos da $\mathrm{TE}^{23,24}$ é que a origem dos EIDs está associada às experiências nocivas ao desenvolvimento emocional de crianças e de adolescentes, incluindo os maus tratos. Estudos prévios têm apontado uma associação entre maus tratos na infância e o desenvolvimento de EIDs, assim como uma influência dos EIDs na manifestação de sintomas psicopatológicos na adolescência ${ }^{13,21,26,27}$. Perpetradores de violência no namoro, com histórico de maus tratos, tiveram escores médios mais al- 
tos nos EIDs do domínio de Desconexão e Rejeição, nos esquemas de arrogo/grandiosidade, busca por aprovação, autocontrole insuficientes, pessimismo e padrões inflexíveis. Tais esquemas podem ser compreendidos tanto pelos modos de enfrentamentos de manutenção, hipercompensação e evitação dos EIDs ${ }^{24,25}$.

O modelo de mediação investigado neste estudo confirmou a hipótese inicial. Os maus tratos na infância foram associados aos EIDs de Desconexão e Rejeição, que por sua vez foram relacionados à perpetração de violência física no namoro. Desta forma, mais do que a exposição aos maus tratos per se, é a associação dos maus tratos com os EIDs do domínio de Desconexão e Rejeição, que se tornam um preditor indireto da perpetração de violência física no namoro. Tal modelo total consegue explicar $15 \%$ da variância da perpetração da violência física no namoro. Estudo prévio ${ }^{29}$, com mulheres vítimas de violência do pareiro íntimo (Turquia), apontou que o domínio de Desconexão e Rejeição mediou a relação entre a exposição aos maus tratos na infância e ser vítima do parceiro íntimo na vida adulta. Em um estudo no Brasil ${ }^{30}$, os EIDs de desconfiança/abuso, defectividade/vergonha, dependência/incompetência, subjugação e isolamento social foram mais fortemente discriminados ao histórico de violência congugal adulta. $\mathrm{Na}$ população de adolescentes perpetradores de violência no namoro, o estudo realizado por Calvete et al. $^{28}$, revelou que o dominínio de Desconexão e Rejeição contribuiu para a associação entre os maus tratos na infância e a violência no namoro na adolescência.

No estudo atual este modelo apresentou variação por sexo, se tornando mais válido para as adolescentes, do que para os adolescentes. A justificativa para esse resultado pode estar associado ao impacto da violência sofrida na infância e nas estratégias cognitivas e emocionais adotadas pelas meninas. Estudos têm indicado que o impacto dos maus tratos e sua relação com a violência no namoro na adolescência pode ser diferente para meninos e meninas, considerando que o processo de socialização e o impacto da exposição à violência intrafamiliar variam de acordo com o gênero $^{14,21}$.

\section{Conclusão}

Os resultados deste estudo apoiam as proposições teóricas referentes à transmissão intergeracional da violência, ampliando sua visão, pois inclui os EIDs como uma variável importante para a compreensão dos mecanismos pelos quais essa transmissão ocorre. Nesse estudo os EIDs do domínio de Desconexão e Rejeição foram caracterizados como mediadores da associação entre os maus tratos na infância e a perpetração de violência física no namoro em adolescentes. Além disso, adolescentes com histórico de maus tratos na infância perpetraram mais violência no namoro, do que adolescentes não vítimasde maus tratos na infância.

Perpetradores de violência no namoro tiveram escores significativamente mais altos nos EIDs do domínio de Desconexão e Rejeição. Indivíduos com esquemas neste domínio acreditam que suas necessidades emocionais básicas de cuidado, segurança, estabilidade dos vínculos afetivos e de pertencimento não serão atendidas ${ }^{24}$. Essas questões estão entrelaçadas às experiências precoces na infância, influenciando a forma como a pessoa percebe, avalia e estabelece suas relações íntimas ${ }^{24}$. Adolescentes que sofreram maus tratos na infância podem carregar consigo os esquemas deste domínio, acreditando que serão abandonados, explorados, humilhados, sendo que a permanência em relações íntimas abusivas pode ser uma forma de evitar o sofrimento emocional associado à ativação de tais EIDs.

Este estudo apresenta algumas limitações. Trata-se de uma pesquisa transversal, com o uso de instrumentos de autorrelato, no qual os adolescentes podem ter respondido de acordo com os padrões de desejabilidade social. Há um viés de sexo na amostra, com um maior número de meninas, o que pode ter influenciado nos resultados. Além disso, meninas se mostram mais abertas para discutir questões íntimas, como situações de violência no namoro. O caráter transversal tem por si só limitações, no que se refere à relação de causalidade entre as variáveis investigadas. Sugere-se pesquisas futuras com delineamento longitudinal e estudos que possam investigar consequências emocionais da exposição dos maus tratos (depressão e estresse pós-traumático, por exemplo) como variáveis moderadoras da violência no namoro. Além disso, estudos futuros podem ainda investigar a associação dos EIDs na formação de crenças legitimadoras da violência ${ }^{14}$ e nos estilos de apego ${ }^{45}$ em adolescentes perpetradores de violência no namoro, que foram expostos aos maus tratos na infância.

Finalmente, os resultados deste estudo podem contribuir para implicações clínicas. Tornam-se necessárias intervenções precoces e de prevenção junto à população jovem, sobretudo 
para aqueles com história de violência na família na infância, por se tornarem um grupo de risco para o ocorrência de violência entre namorados. Intervenções clínicas, dentro da abordagem da Terapia do Esquema, podem utilizar estratégias de imagens mentais, da reparentalização limitada e da reestruturação cognitiva ${ }^{24}$, a fim de modificar esquemas disfuncionais.

\section{Colaboradores}

JL Borges trabalhou na concepção da pesquisa, coleta, análise e interpretação dos dados e redação do artigo. DD Dell'Aglio trabalhou na concepção da pesquisa, análise dos dados e na revisão final do artigo.

\section{Agradecimentos}

Ao Conselho Nacional de Desenvolvimento Científico e Tecnológico (CNPq) e à Coordenação de Aperfeiçoamento de Pessoal de Nível Superior (CAPES), pelo apoio financeiro. 


\section{Referências}

1. Mulford CE, Blachman-Demner DR. Teen dating violence: Building a research program through collaborative insights. Violence Against Women 2013; 19(6):756-770.

2. Wolfe DA, Scott K, Reitzel-Jaffe D, Wekerle C. Development and validation of the conflict in adolescent dating relationships inventory. Psychol Assess 2011; 13(2):277-293

3. Beserra MA, Leitão MNC, Fabião JASAO, Dixe MACR, Veríssimo CMF, Ferriani MGC. Prevalência e características da violência no namoro entre adolescentes escolares de Portugal. Esc Anna Nery 2016; 20(1):183-191.

4. Jennings WG, Park M, Richards TN, Tomsich E, Gover A, Powers RA. Exploring the relationship between child physical abuse and adult dating violence using a causal inference approach in an emerging adult population in South Korea. Child Abuse Negl 2014; 38(12):1902-1913.

5. Fernández-Fuertes AA, Fuertes A. Physical and psychological aggression in dating relationships of Spanish adolescents: Motives and consequences. Child Abuse Negl 2010; 34(3):183-191.

6. Straus MA. Prevalence of violence against dating partners by male and female university students worldwide. Violence Against Women 2004; 10(7):790-811.

7. Wincentak K, Connolly J, Card N. Teen dating violence: A meta-analytic review of prevalence rates. Psychol Violence 2017; 7:224-241.

8. Choi HJ, Weston R, Temple JR. A three-step latent class analysis to identify how different patterns of teen dating violence and psychosocial factors influence mental health. J Youth Adolesc 2017; 46(4):854-866.

9. Lazarevich I, Camacho MEI, Sokolova AV, Gutiérrez HJD. Violencia en el noviazgo y salud mental en estudiantes universitários mexicanos. Glob Health Promot 2013; 20(3):94-103.

10. Oliveira QBM, Assis SG, Njaine K, Oliveira RVC. Violências nas relações afetivo-sexuais. In: Minayo MCS, Assis SG, Njaine K, editores. Amor e violência: Um paradoxo das relações de namoro e do "ficar" entre jovens brasileiros. Rio de Janeiro: Fiocruz; 2011. p. 87-139.

11. Faias J, Caridade $\mathrm{S}$, Cardoso J. Exposição à violência familiar e abuso íntimo em jovens: Que relação? Psychologica 2016; 59(1):7-23.

12. Gómez AM. Testing the cycle of violence hypothesis: Child abuse and adolescent dating violence as predictors of intimate partner violence in young adulthood. Youth Society 2011; 43:171-192.

13. Izaguirre A, Calvete E. Exposure to family violence as a predictor of dating violence and child-to-parent aggression in Spanish adolescents. Youth Society 2017; 49(3):393-412.

14. Wolfe DA, Wekerle C, Scott K, Straatman A, Grasley C. Predicting abuse in adolescent dating relationship over 1 year: The role of child maltreatment and trauma. J Abnorm Psychol 2004; 113:406-415.

15. Kaukinen C, Buchanan L, Gover AR. Child abuse and experience of violence in college dating relationships: Examining the moderating effect of gender and race. $J$ Fam Violence 2015; 30(8):1079-1092.

16. Bandura A. Aggression: A social learning analysis. Englewood Cliffs, New Jersey: Prentice-Hall; 1973.
17. Caridade S. Vivências intimas violentas: Uma abordagem científica. Portugal/Coimbra: Almedina; 2011.

18. Ponce AN, Williams MK, Allen GJ. Experience of maltreatment as a child and acceptance of violence in adult intimate relationships: Mediating effects of distortions in cognitive schemas. Violence Vict 2004; 19(1):97-108.

19. Clarey A, Hokoda A, Ulloa EC. Anger control and acceptance of violence as mediators in the relationship between exposure to interparental conflict and dating violence perpetration in Mexican adolescents. J Fam Violence 2010; 25(7):619-625.

20. Reyes HLM, Foshee VA, Fortson BL, Valle LA, Breiding MJ, Merrick MT. Longitudinal mediators of relations between family violence and adolescent dating aggression perpetration. J Marriage Fam 2015; 77(7):1016-1030.

21. Calvete E, Orue I. Cognitive mechanisms of the transmission of violence: Exploring gender differences among adolescents exposed to family violence. J Fam Violence 2013; 28(1):73-84.

22. Gover AR, Park M, Tomsich EA, Jennings WG. Dating violence perpetration and victimization among South Korean college students: A focus on gender and childhood maltreatment. J Interpers Violence 2011; 26(6):1232-1263.

23. Young JE. Terapia cognitiva para transtornos de personalidade: Uma abordagem focada no esquema. $3^{\mathrm{a}} \mathrm{ed}$. Porto Alegre: Artmed; 2003.

24. Young J, Klosko JS, Weishaar ME. Terapia do esquema: Guia de técnicas cognitivo-comportamentais inovadoras. Porto Alegre: Artmed; 2008.

25. Wainer R, Rijo D. O modelo teórico: Esquemas iniciais desadaptativos, estilos de enfrentamento e modos esquemáticos. In: Wainer R, Paim K, Erdos R, Andriola R, editores. Terapia cognitiva focada em esquemas. Porto Alegre: Artmed; 2016. p. 47-63.

26. Calvete E. Emotional abuse as a predictor of early maladaptive schemas in adolescents: Contributions to the development of depressive and social anxiety symptoms. Child Abuse Negl 2014; 38(4):735-746.

27. Wright MO, Crawford E, Del Castillo D. Childood emotional maltreatment and later psychological distress among college students: The mediating role of maladaptive schemas. Child Abuse Negl 2009; 33(1):59-68.

28. Calvete E, Fernández-González L, Orue I, Little TD. Exposure to Family violence and dating violence perpetration in adolescents: Potential cognitive and emotional mechanisms. Psychol Violence 2018; 8(1):67-75.

29. Atmaca S, Gençöz T. Exploring revictimization process among Turkish women: The role of early maladaptive schemas on the link between child abuse and partner violence. Child Abuse Negl 2016; 52:85-93.

30. Paim K, Falcke D. Perfil discriminane de sujeitos com histórico de violência conjugal: $\mathrm{O}$ papel dos esquemas iniciais desadaptativos. Rev Bras Ter Comport Cogn 2016; XVIII(2):112-129.

31. Taskale N, Soygüt G. Risk factors for women's intimate partner violence victimization: An examination from the perspective of the Schema Therapy Model. J Fam Violence 2017; 32(1):3-12. 
32. Paim K, Madalena M, Falcke D. Esquemas iniciais desadaptativos na violência conjugal. Rev Bras Ter Cogn 2012; 8(1):31-39.

33. Barreira AK, Lima MLC, Avanci JQ. Coocorrência de violência física e psicológica entre adolescentes do Recife, Brasil: Prevalência e fatores associados. Cien Saude Colet 2013; 18(1):233-243.

34. Oliveira QBM, Assis SG, Njaine K, Pires TO. Namoro na adolescência no Brasil: Circularidade da violência psicológica nos diferentes contextos relacionais. Cien Saude Colet 2014; 19(3):707-718.

35. Avanci JQ, Assis SG. Opções metodológicas. In: Minayo MSC, Assis SG, Njaine K, editores. Amor e violência: Um paradoxo das relações de namoro e do 'ficar' entre jovens brasileiros. Rio de Janeiro: Fiocruz; 2011. p. 302-314.

36. Santos L, Vagos P, Rijo D. Dimensionality and Measurement Invariance of a Brief Form of the Young Schema Questionnaire for Adolescents. J Child Fam Stud 2018; 27(1):1-12.

37. Borges JL, Vagos P, Dell'Aglio DD, Rijo D. Cross-cultural validation of the Young Schema Questionnaire for Adolescents in Portuguese and Brazilian samples. Int J Cogn Ther [periódico na Internet]. 2020; [first online version]. Disponível em: https://doi.org/10.1007/ s41811-020-00067-6.

38. Borges JL, Dell'Aglio DD. Abuso sexual infantil: Indicadores de risco e consequências no desenvolvimento de crianças. Interam J Psicol 2008; 42(3):528-536.

39. Brasil. Ministério da Saúde (MS). Secretaria de Políticas de Saúde. Violência intrafamiliar: orientações para prática em serviço. Série Cadernos de Atenção Básica 8. Brasília: MS; 2001.

40. Cohen J. Statistical power analysis for the behavioral sciences. $2^{\text {a }}$ ed. Hillsdale: Erlbaum; 1998.

41. Baron RM, Kenny DA. The moderator-mediator variable distinction in social psychological research: Conceptual, strategic, and statistical considerations. J Pers Soc Psychol 1986; 51(6):1173-1182.

42. Rosseel Y. Lavaan: An R package for structural equation modeling. J Statistical Software 2012; 48:1-36.

43. R Development Core Team. $R$ Foundation for Statistical Computing. Vienna, Austria; 2011.

44. Hair JF Jr, Black WC, Babin BJ, Anderson RE, Tatham RL. Análise multivariada de dados. $6^{\mathrm{a}}$ ed. Porto Alegre: Bookman; 2009.

45. Lee M, Reese-Weber M, Kahn JH. Exposure to family violence and attachment styles as predictors of dating violence perpetration among men and women: A mediational model. J Interpers Violence 2014; 29(1):2043.

Artigo apresentado em 05/06/2018

Aprovado em 20/11/2018

Versão final apresentada em 22/11/2018 